\title{
Stochastic Finite Integration Technique Formulation for Electrokinetics
}

\author{
Lorenzo Codecasa and Luca Di Rienzo \\ Dipartimento di Elettronica, Informazione e Bioingegneria, Politecnico di Milano, I-20133, Milan, Italy \\ codecasa@elet.polimi.it, luca.dirienzo@polimi.it
}

\begin{abstract}
A stochastic finite integration technique formulation of an electrokinetic problem is derived applying the polynomial chaos expansion. The formulation is able to provide the uncertainty quantification of fields and integral quantities. It is applied to a test case representing an industrial application and the obtained results are in good agreement with those calculated by means of Monte Carlo simulations, with the advantage of a dramatic reduction of the computational time.
\end{abstract}

Index Terms-Electrokinetics, Finite Integration Technique, Polynomial Chaos Expansion.

\section{INTRODUCTION}

Electromagnetic computations rely on the perfect knowledge of material parameters. However, for a wide range of examples in electrical engineering, some uncertainty should be associated with that knowledge in the modeling process. In order to quantify the uncertainty of the output quantities of interest coming from the lack of knowledge of the input material parameters, the Monte Carlo (MC) method can be applied. However the convergence of the estimates of the statistical moments versus the number of required deterministic simulations is slow and, if the single deterministic computation is not fast enough, the total computational time becomes prohibitive. The spectral stochastic finite element method based on the polynomial chaos expansion (PCE) is introduced in [1] and is able to dramatically reduce the computational time in many electromagnetic problems, e.g. [2]. In the present paper for the first time the PCE is formulated for the finite integration technique (FIT) [3], and the specific structure of the resulting stochastic FIT formulation is determined

The prototype problem under analysis is of electrokinetics type. More specifically, in order to test the formulation, a typical system for resistance welding is analyzed [4].

\section{Deterministic FIT Formulation}

The stochastic FIT formulation here proposed starts from a deterministic FIT formulation, derived by discretizing the spatial region of the problem $\Omega$ by a pair of three-dimensional oriented dual grids, $\mathcal{G}$ and $\tilde{\mathcal{G}}$. In order to manage boundary conditions in a general way, unlike standard FIT, the pair of two-dimensional oriented dual grids, $\mathcal{G}_{b}$ and $\tilde{\mathcal{G}}_{b}$ are also introduced, where $\mathcal{G}_{b}$ is the trace of the primal grid $\mathcal{G}$ onto the boundary $\partial \Omega$ of $\Omega$ and $\tilde{\mathcal{G}}_{b}$ is its dual [7]. The continuity law for currents is discretized, in exact form, as follows

$$
\tilde{\mathbf{D}} \tilde{\boldsymbol{i}}+\tilde{\mathbf{D}}_{b} \tilde{\boldsymbol{i}}_{b}=\mathbf{0},
$$

in which $\tilde{\boldsymbol{i}}$ and $\tilde{\boldsymbol{i}}_{b}$ are the vectors of the fluxes of the electric current through the faces of $\tilde{\mathcal{G}}$ and $\tilde{\mathcal{G}}_{b}, \tilde{\mathbf{D}}$ is the volume-face incidence matrix of $\tilde{\mathcal{G}}, \tilde{\mathbf{D}}_{b}$ is the incidence matrix between the volumes of $\tilde{\mathcal{G}}$ and the faces of $\tilde{\mathcal{G}}_{b}$. The irrotationality of the electric field is expressed, in exact form, as

$$
\boldsymbol{v}=-\mathbf{G} \boldsymbol{\varphi},
$$

in which $v$ is the vector with the circulations of the electric field along the edges of $\mathcal{G}, \varphi$ is the vector of the electric potentials at the nodes of $\mathcal{G}$ and $\mathbf{G}$ is the edge-node incidence matrix of $\mathcal{G}$. As it is well known, for any pair of dual grids $\mathbf{G}^{T}=-\tilde{\mathbf{D}}$. Ohm's law is discretized in approximate form, in terms of a discrete material matrix $\mathbf{M}_{\sigma}$ as follows

$$
\tilde{\boldsymbol{i}}=\mathbf{M}_{\sigma} \boldsymbol{v}
$$

The discrete material matrix $\mathbf{M}_{\sigma}$, can be derived for generic polyhedral primal grids $\mathcal{G}$, using the energetic approach introduced in [5] for a tetrahedral grid and extended in [6] for generic polyhedral grids. Lastly boundary conditions are introduced. For a Dirichlet problem, here considered, such conditions can be written in exact form in terms of the incidence matrix $\tilde{\mathbf{D}}_{b}$ as follows [7]

$$
\tilde{\mathbf{D}}_{b}^{T} \boldsymbol{\varphi}=\boldsymbol{\varphi}_{b},
$$

in which $\varphi_{b}$ is the vector of the electric potential on the boundary $\partial \Omega$, hence assumed known.

\section{Stochastic FIT Formulation}

The electric conductivity is now assumed to be a spatial field function of a random event $\vartheta$ and thus can be written as $\sigma(\mathbf{r}, \vartheta), \mathbf{r}$ being the position vector. PCEs can now be introduced for the electric potential, the electric field, and the current density. From these expansions analogous expansions follows for the discrete variables of FIT. Precisely, it is

$$
\boldsymbol{v}(\vartheta)=\sum_{\boldsymbol{\alpha}} \boldsymbol{v}_{\boldsymbol{\alpha}} \psi_{\boldsymbol{\alpha}}(\vartheta)
$$

in which $\psi_{\boldsymbol{\alpha}}(\vartheta)$ are the chosen polynomials with multi indices $\boldsymbol{\alpha}$, supposed to be orthonormal in a chosen probabilistic space so that $\mathbf{E}\left[\psi_{\boldsymbol{\alpha}}(\vartheta) \psi_{\boldsymbol{\beta}}(\vartheta)\right]=\delta_{\boldsymbol{\alpha} \boldsymbol{\beta}}$, in which $\mathbf{E}[\cdot]$ indicates the statistical mean value. In this way $\boldsymbol{v}_{\boldsymbol{\alpha}}$ assumes the meaning of the projection of the stochastic variable $\boldsymbol{v}(\vartheta)$ onto $\psi_{\boldsymbol{\alpha}}(\vartheta)$ so that $\boldsymbol{v}_{\boldsymbol{\alpha}}=\mathbf{E}\left[\boldsymbol{v}(\vartheta) \psi_{\boldsymbol{\alpha}}(\vartheta)\right]$. In a similar way PCE is introduced for the other variables $\tilde{\boldsymbol{i}}(\vartheta), \tilde{\boldsymbol{i}}_{b}(\vartheta), \varphi(\vartheta), \boldsymbol{\varphi}_{b}(\vartheta)$. Substituting these expansions of the discrete variables, assumed stochastic, 
into the exact balance equations (1), (2) and projecting in the stochastic space onto each polynomial $\psi_{\boldsymbol{\alpha}}(\vartheta)$ the stochastic equations follow. In fact from (1) it ensues

$$
\mathbf{E}\left[\psi_{\boldsymbol{\alpha}}(\vartheta)\left(\tilde{\mathbf{D}} \tilde{\boldsymbol{i}}(\vartheta)+\tilde{\mathbf{D}}_{b} \tilde{\boldsymbol{i}}_{b}(\vartheta)\right)\right]=\mathbf{0}
$$

so that

$$
\tilde{\mathbf{D}} \tilde{\boldsymbol{i}}_{\boldsymbol{\alpha}}+\tilde{\mathbf{D}}_{b} \tilde{\boldsymbol{i}}_{b \boldsymbol{\alpha}}=\mathbf{0} .
$$

In a similar way, from (2) it follows

$$
v_{\alpha}=-\mathbf{G} \varphi_{\alpha} .
$$

As a result all the balance equations are left unaltered after projection in the probabilistic space. All these equations can be re-written in the following forms

$$
\begin{aligned}
& \left(\mathbf{I}_{n} \otimes \tilde{\mathbf{D}}\right) \tilde{\mathbf{I}}+\left(\mathbf{I}_{n} \otimes \tilde{\mathbf{D}}_{b}\right) \tilde{\mathbf{I}}_{b}=\mathbf{0}, \\
& \mathbf{V}=-\left(\mathbf{I}_{n} \otimes \mathbf{G}\right) \boldsymbol{\Phi},
\end{aligned}
$$

in which $n$ is the number of polynomials $\psi_{\boldsymbol{\alpha}}(\vartheta)$ in the PCE, $\otimes$ indicates tensor product, $\tilde{\mathbf{I}}$ is the vector constituted by subvectors $\tilde{\boldsymbol{i}}_{\boldsymbol{\alpha}}$ and $\tilde{\mathbf{I}}_{b}$ is the vector constituted by sub-vectors $\tilde{\boldsymbol{i}}_{b \boldsymbol{\alpha}}$. In similar way $\mathbf{V}$ is the vector constituted by sub-vectors $\boldsymbol{v}_{\boldsymbol{\alpha}}$ and $\boldsymbol{\Phi}$ is the vector constituted by sub-vectors $\varphi_{\alpha}$. It is noted that $\left(\mathbf{I}_{n} \otimes \mathbf{G}\right)^{T}=-\left(\mathbf{I}_{n} \otimes \tilde{\mathbf{D}}\right)$. By projecting (3) in the stochastic space onto each polynomial $\psi_{\boldsymbol{\alpha}}(\vartheta)$ it follows

$$
\tilde{\mathbf{I}}=\mathbf{N}_{\sigma} \mathbf{V}
$$

in which $\mathbf{N}_{\sigma}$ is a block matrix whose $\boldsymbol{\alpha} \boldsymbol{\beta}$-th block $\mathbf{N}_{\sigma \boldsymbol{\alpha} \boldsymbol{\beta}}$ is an $l$-order matrix, which is the discrete constitutive matrix of a deterministic problem in which the spatial distribution of conductivity is substituted by the spatial distribution $\mathbf{E}\left[\sigma(\mathbf{r}, \vartheta) \psi_{\boldsymbol{\alpha}}(\vartheta) \psi_{\boldsymbol{\beta}}(\vartheta)\right]$. Lastly boundary conditions can be discretized in the form

$$
\tilde{\mathbf{D}}_{b}^{T} \boldsymbol{\varphi}_{\boldsymbol{\alpha}}=\boldsymbol{\varphi}_{b \boldsymbol{\alpha}} .
$$

As a result all the boundary conditions equations are left unaltered after projection in the probabilistic space. All these equations can be re-written in the following forms

$$
\left(\mathbf{I}_{n} \otimes \tilde{\mathbf{D}}_{b}\right)^{T} \boldsymbol{\Phi}=\boldsymbol{\Phi}_{b},
$$

in which $\boldsymbol{\Phi}_{b}$ is the vector constituted by sub-vectors $\varphi_{b \boldsymbol{\alpha}}$. It is thus noted that only the discrete material equations have to be modified in the stochastic formulation with respect to the deterministic formulation.

\section{NumericAl Results}

The 3D problem shown in Fig. 1 is considered. Three electrodes over a conductive substrate exhibit contact resistances modeled by three statistically independent conductivities $\sigma_{1}$, $\sigma_{2}, \sigma_{3}$, with uniformly distributed probability density functions in the range $1.41 \mathrm{MS} / \mathrm{m}-11.3 \mathrm{MS} / \mathrm{m}$.

A tetrahedral grid is generated, with $n=5,081$ nodes, whose further refining introduces variations in the potential distribution lesser that $1 \%$ in the energy norm. A FIT discretization is introduced, using the basis functions introduced in [5] for tetrahedral grids. The PCE of the electric current $I_{1}$

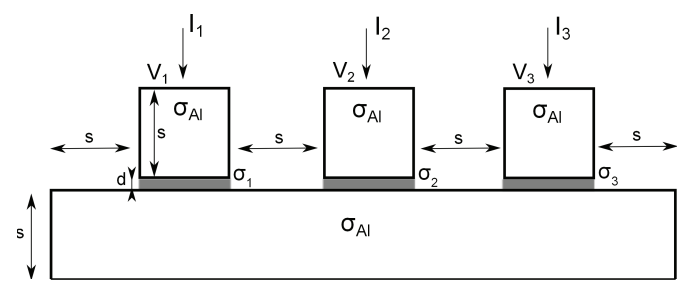

Figure 1. 2D section of the considered example, the depth being equal to $s$, with $s=10 \mathrm{~mm}$ and $d=1 \mathrm{~mm}$.

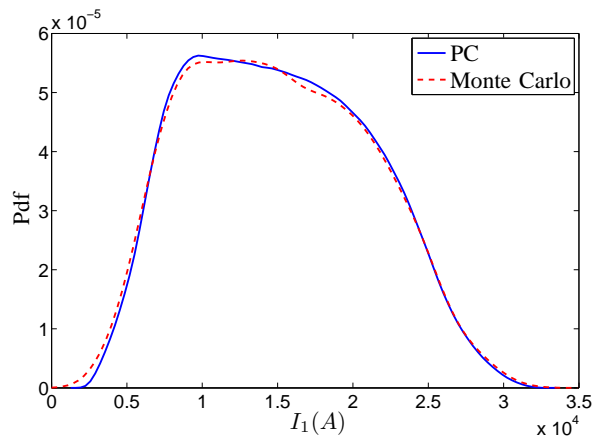

Figure 2. Probability density function of the current $I_{1}$.

is estimated in the situation in which $V_{1}=V_{3}=1$ and $V_{2}=0$. The variation of the probability density function (pdf) of $I_{1}$ for an increase of the number $p$ of polynomials used for each independent random variable in the PCE, maintains below $1 \%$ when $\mathrm{p}$ is greater than 3 . For $p=4$ the pdf of the current $I_{1}$ assumes the form shown in Fig. 2. The solution of the system of equations is performed by a proper iterative solver, a preconditioned conjugate gradient algorithm, and takes about 31 seconds on a $2.3 \mathrm{Ghz}$ Intel Core i7. The accuracy of the results can be checked by a Monte Carlo method, by repeating the deterministic FIT simulation with $10^{5}$ random choices of the random variables. This computations require about 10 hours. The pdf obtained with stochastic FIT is in agreement with the pdf given by Monte Carlo simulations with an error below $5 \%$.

\section{REFERENCES}

[1] R. Ghanem, P. Spanos, Stochastic Finite Elements: A Spectral Approach, World Publishing Corporation, 1991.

[2] K. Beddek, Y. Le Menach, S. Clenet, and O. Moreau, "3-D Stochastic Spectral Finite-Element Method in Static Electromagnetism Using Vector Potential Formulation," IEEE Transactions on Magnetics, Vol. 47, No. 5, pp. 1250-1253, 2011.

[3] H. De Gersem, I. Munteanu, T. Weiland, "Construction of Differential Material Matrices for the Orthogonal Finite-Integration Technique With Nonlinear Materials," IEEE Transactions on Magnetics, Vol. 44, No. 6, pp. $710-713,2008$.

[4] S. Clenet and N. Ida, "Error estimation in a stochastic finite element method in electrokinetics," International Journal for Numerical Methods in Engineering, Vol. 81, No. 11, pp. 1417-1438, 2010.

[5] L. Codecasa, V. Minerva, M. Politi, "Use of barycentric dual grids for the solution of frequency domain problems by FIT," IEEE Transactions on Magnetics, Vol. 40, No. 2, pp. 1414-1419, 2004

[6] L. Codecasa, F. Trevisan, "Constitutive equations for discrete electromagnetic problems over polyhedral grids," Journal of Computational Physics, Vol. 225, No. 2, pp. 1894-1918, 2007.

[7] L. Codecasa, "Refoundation of the Cell Method by means of Augmented Dual Grids," accepted at COMPUMAG 2013. 